\title{
Phase transfer of platinum nanoparticles from aqueous to organic solutions using fatty amine molecules
}

\author{
ASHAVANI KUMAR, ${ }^{1}$ HRUSHIKESH M JOSHI, ${ }^{1}$ ANANDRAO B MANDALE, ${ }^{2}$ \\ RAJENDRA SRIVASTAVA, ${ }^{3}$ SUGUNA D ADYANTHAYA, ${ }^{1}$ RENU PASRICHA ${ }^{2}$ and \\ MURALI SASTRY ${ }^{1 *}$ \\ ${ }^{1}$ Materials Chemistry Division, ${ }^{2}$ Centre for Materials Characterisation, and ${ }^{3}$ Catalysis Division, National \\ Chemical Laboratory, Pune 411 008, India \\ e-mail: sastry@ems.ncl.res.in
}

MS received 4 March 2004; revised 29 July 2004

\begin{abstract}
In this report we demonstrate a simple process based on amine chemistry for the phase transfer of platinum nanoparticles from an aqueous to an organic solution. The phase transfer was accomplished by vigorous shaking of a biphasic mixture of platinum nanoparticles synthesised in an aqueous medium and octadecylamine (ODA) in hexane. During shaking of the biphasic mixture, the aqueous platinum nanoparticles complex via either coordination bond formation or weak covalent interaction with the ODA molecules present in the organic phase. This process renders the nanoparticles sufficiently hydrophobic and dispersible in the organic phase. The ODA-stabilised platinum nanoparticles could be separated out from hexane in the form of a powder that is readily redispersible in weakly polar and nonpolar organic solvents. The ODA-capped platinum nanoparticles show high catalytic activity in hydrogenation reactions and this is demonstrated in the efficient conversion of styrene to ethyl benzene. The nature of binding of the ODA molecules to the platinum nanoparticles surface was characterised by thermogravimetry, transmission electron microscopy (TEM), X-ray photoemission spectroscopy (XPS) and Fourier transform infrared spectroscopy (FTIR).
\end{abstract}

Keywords. Nanoparticles; platinum; phase transfer; hydrophobization; X-ray photoemission spectroscopy.

\section{Introduction}

Metal nanoparticles have important applications in the area of catalysis, ${ }^{1,2}$ electronics, ${ }^{3}$ as electron microscopy markers, ${ }^{4}$ in DNA detection ${ }^{5}$ etc. due to their size and shape-dependent optical and electronic properties. Consequently, a number of strategies have been developed to synthesise metal nanoparticles in polar (water based) ${ }^{6-13}$ and nonpolar (organic) ${ }^{14-17}$ solvents because the colloidal stability, particle size, and its properties depend strongly on the specific method of preparation. Water-based synthesis protocols include reduction of aqueous metal ions by electromagnetic radiation, ${ }^{9,10}$ sonochemistry $^{11}$ and the popular chemical reducing agents. ${ }^{6-8}$ One of the main drawbacks of water-based metal nanoparticle synthesis protocols is the poor control over the size and monodispersity of nanoparticles that can be realised and, furthermore, the nanoparti-

\footnotetext{
$\overline{\text { *For correspondence }}$
}

cles are not re-dispersible in water after extraction from the aqueous phase. ${ }^{18,19}$ Ionic effects limit the concentration of nanoparticles that can be synthesised in water, which can be a significant drawback in biological applications where high nanoparticle concentrations are desirable. ${ }^{5}$

Brust and co-workers have developed a method for the synthesis of gold nanoparticles in non-polar organic solvents which involves extraction of metal ions such as chloroaurate ions into toluene using a phase transfer molecule such as tetra-alkyl ammonium bromide. ${ }^{14}$ Thereafter, reduction of metal ions using sodium borohydride in the presence of capping agents, such as thiol and alkylamine molecules resulted in the formation of monodisperse nanoparticles of desired size that can be extracted from solution in the form of a powder without significant variation in the particle size distribution. In principle, very high concentrations of metal nanoparticles may be readily synthesised in organic solutions, thus overcoming an important drawback associated with 
water-based synthesis. An added advantage of organic solution-based synthesis protocols is that the nanoparticles spontaneously assemble into hexagonal close-packed monolayers on suitable surfaces during solvent evaporation. ${ }^{14}$ On the other hand, one of the major disadvantages of the Brust method is that it is a multistep one and therefore time-consuming.

It is clear that both water and organic solutionbased nanoparticle synthesis methods possess characteristic advantages and disadvantages. It should be possible to maximise the advantages of the two protocols by combining them in some way. This may be easily achieved by synthesising the nanoparticles in one medium and, thereafter, transferring them to the complementary phase. A number of experimental protocols exist in the literature for the phase transfer of nanoparticles from aqueous to organic phases. ${ }^{20-27}$ Sarathy et al have demonstrated that colloidal gold, platinum and silver particles first synthesized in water may be transferred into a non-polar environment such as toluene by acid-facilitated co-ordination of the particles with thiols present in toluene. ${ }^{21,22}$ This method has also been applied to the acid-facilitated phase transfer of oleate-stabilised colloidal silver particles by Wang, Efrima and Regev. ${ }^{23}$ In addition to alkanethiols for surface modification of gold, silver and platinum nanoparticles, a small number of reports have appeared in the literature on surfacemodification of gold nanoparticles using alkyamines. ${ }^{28,29}$ Some of us have shown recently that complexation of alkylamine molecules with aqueous gold nanoparticles at a liquid-liquid interface may be used as an alternative route to gold nanoparticle phase transfer from water to organic solvents. ${ }^{25}$

Pt nanoparticles are of great interest because of their excellent catalytic activity. ${ }^{30-32}$ To prepare stable Pt nanoparticles, polymers ${ }^{30}$ and polyelectrolytes $^{31-33}$ are used as protective matrixes. Ahmadi et $a l^{33}$ have demonstrated that Pt nanoparticles of different shapes can be produced by changing the concentration of protector (sodium polyacrylate) in solution. In a previous study, we have reported the phase transfer of colloidal gold nanoparticles synthesized in aqueous phase, into different organic solvents by using octadecylamine molecules as the phase transfer agent. ${ }^{25}$ Here we extend our study in this direction and demonstrate that colloidal platinum particles synthesised in water can be quantitatively transferred into hexane by direct co-ordination with octadecylamine molecules present in the organic phase. Vigorous shaking of the biphasic mixture
(Pt-in-water/ODA-in-hexane) results in the rapid complexation of ODA molecules with Pt nanoparticles and, thereafter, a swift transfer of the now hydrophobic ODA-stabilised Pt nanoparticles into the organic phase. Close-packed Pt nanoparticle films are easily prepared by simple immersion of suitable substrates in the nanoparticle solution and evaporation of the organic component. The hydrophobized colloidal platinum particles show high catalytic activity and may be repeatedly dried and redispersed in different organic solvents. The Pt nanoparticles at various stages of the phase-transfer and film formation process have been characterised using Fourier transform infrared (FTIR) spectroscopy, thermogravimetry (TGA), X-ray photoemmision spectroscopy (XPS) and transmission electron microscopy (TEM). Presented below are details of the investigation.

\section{Experimental section}

In a typical experiment, platinum nanoparticles were synthesised by borohydride reduction of aqueous $\mathrm{H}_{2} \mathrm{PtCl}_{6}$ solution $\left(10^{-4} \mathrm{M}\right.$ of aqueous solution of $\mathrm{H}_{2} \mathrm{PtCl}_{6}$ ) in a manner analogous to that described elsewhere for gold nanoparticles. ${ }^{34}$ This results in a clear blackish-brown colloidal solution at $\mathrm{pH} 9$ containing platinum particles of $10 \pm 2 \mathrm{~nm}$ average size. To $100 \mathrm{ml}$ of the platinum colloidal solution thus prepared, $100 \mathrm{ml}$ of a $2 \times 10^{-4} \mathrm{M}$ solution of octadecylamine (ODA) in hexane was added to yield immiscible layers of the colourless organic solution on top of the brown-coloured platinum hydrosol (figure 1A). Vigorous shaking of the test-tube resulted in transfer of the platinum colloidal particles into the organic phase and this was observed by the brown colouration of the organic phase (and a corresponding loss of colour from the aqueous phase) when the two layers separated out (figure 1B). As mentioned earlier, this occurs spontaneously and did not require addition of acid, which is an essential ingredient in the alkanethiol coupled phase transfer protocol. The ODA-capped platinum nanoparticles were separated out by rotavapping the organic phase as a dry powder and purified by repeated washing with ethanol. The process of washing with ethanol removes uncoordinated ODA molecules from the platinum nanoparticle powder.

The purified ODA-Pt nano powder was redispersed in chloroform and characterised by UV-Vis spectroscopy, Fourier transform infrared spectroscopy (FTIR), thermo-gravimetric analysis (TGA), 
X-ray photoemission spectroscopy (XPS) and transmission electron spectroscopy (TEM). UV-Vis spectra of the platinum nanoparticle solutions were recorded on a Shimadzu dual-beam spectrophotometer (model UV-1601 PC) operated at a resolution of $1 \mathrm{~nm}$. Drop-coated films of the platinum nanoparticles on Si (111) substrates were analysed by FTIR and X-ray photoelectron spectroscopy. FTIR measurements were carried out in the diffuse reflectance mode on a Perkin-Elmer Spectrum 1 instrument operated at a resolution of $2 \mathrm{~cm}^{-1}$. XPS measurements on the platinum nanoparticle film were carried out on a VG MicroTech ESCA 3000 instrument at a pressure of better than $1 \times 10^{-9}$ Torr. The general scan and $\mathrm{C} 1 s$, Pt $4 f$ and $\mathrm{N} 1 s$ core level spectra were recorded with un-monochromatized $\mathrm{MgK}_{\alpha}$ radiation (photon energy $=1253.6 \mathrm{eV}$ ) at a pass energy of $50 \mathrm{eV}$ and electron takeoff angle (angle between electron emission direction and surface plane) of $60^{\circ}$. The overall resolution was $\sim 1 \mathrm{eV}$ for the XPS measurements. The core level spectra were background corrected using the Shirley algorithm ${ }^{35}$ and the chemically distinct species resolved using a nonlinear least squares fitting procedure. The core level binding energies (BEs) were aligned with the adventitious carbon binding energy of $285 \mathrm{eV}$. TGA analysis of the purified platinum nanoparticle powder was done on a TGA-7 Perkin-Elmer instrument in

(A)

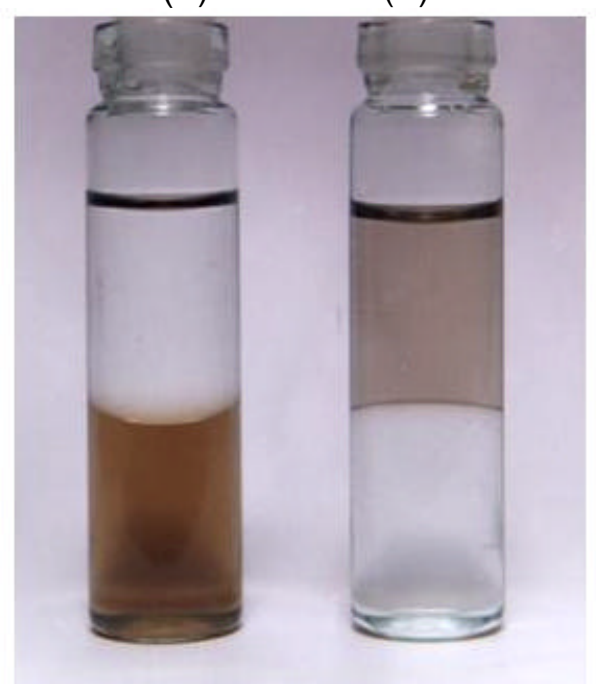

Figure 1. (A) Immiscible layers of the platinum hydrosol (layer at the bottom) and the clear hexane solution containing ODA (on top) before shaking. (B) Alkylamine-derivatized $\mathrm{Pt}$ nanoparticles in hexane (layer on top) and the clear aqueous solution at the bottom after the phase transfer by shaking the biphasic mixture. the temperature range $0-800^{\circ} \mathrm{C}$ at a scan rate of $10^{\circ} \mathrm{C} / \mathrm{min}$. TEM measurements of drop-coated films of the platinum nanoparticles on carbon-coated $\mathrm{Cu}$ TEM grids were performed on a JEOL Model $1200 \mathrm{EX}$ instrument operated at an accelerating voltage of $120 \mathrm{kV}$.

\section{Results and discussion}

The phase transfer of the aqueous platinum nanoparticles into the organic phase is illustrated in figure 1, which shows pictures of a test-tube before (figure 1A) and after (figure 1B) phase transfer of the colloidal Pt particles into hexane. The phase transfer was observed by the brown colouration of the organic phase and a corresponding almost complete loss of colour from the aqueous phase when the two layers separated out (figure 1B). After phase transfer, the organic phase was separated out from the mixture and characterised by TEM.

Drop-coated films of the ODA-capped platinum nanoparticles were formed on carbon-coated copper grids by solvent evaporation for transmission electron microscopy (TEM) measurements. Figure 2 shows representative TEM images of ODA-capped platinum nanoparticles in hexane before (A and $\mathrm{B}$ ) and after the hydrogenation reaction (C and $\mathrm{D})$ at low and high magnifications respectively. It is clear from the TEM picture that the grid is uniformly covered with extremely large domains of platinum nanoparticles. The higher magnification image reveals the close packing of the platinum nanoparticles (figure 2B). While some degree of sintering of the particles appears to have occurred, particles in the 2-D assembly are to a large extent well separated from one another. Figure 3A shows the histogram of the particle size distribution measured from particles in figure $2 \mathrm{~B}$ and other similar micrographs. A Gaussian fit to the histogram yielded an average particle size of $10 \pm 2 \mathrm{~nm}$. Figure 3B shows the electron diffraction pattern measured from the platinum nanoparticles of figure $2 \mathrm{~B}$. It is clear from the electron diffraction pattern that the nanoparticles are crystalline; the diffraction rings could be indexed on the basis of the $f c c$ structure of platinum. Comparison of the TEM images recorded from the ODA-capped Pt nanoparticles before (figures $2 \mathrm{~A}$ and $\mathrm{B}$ ) and after hydrogenation reactions (figures $2 \mathrm{C}$ and $\mathrm{D}$ ) show that there is no detectable change in the average particle size after reaction. The fact that the particles are not aggregated after hydrogenation reaction also indica- 

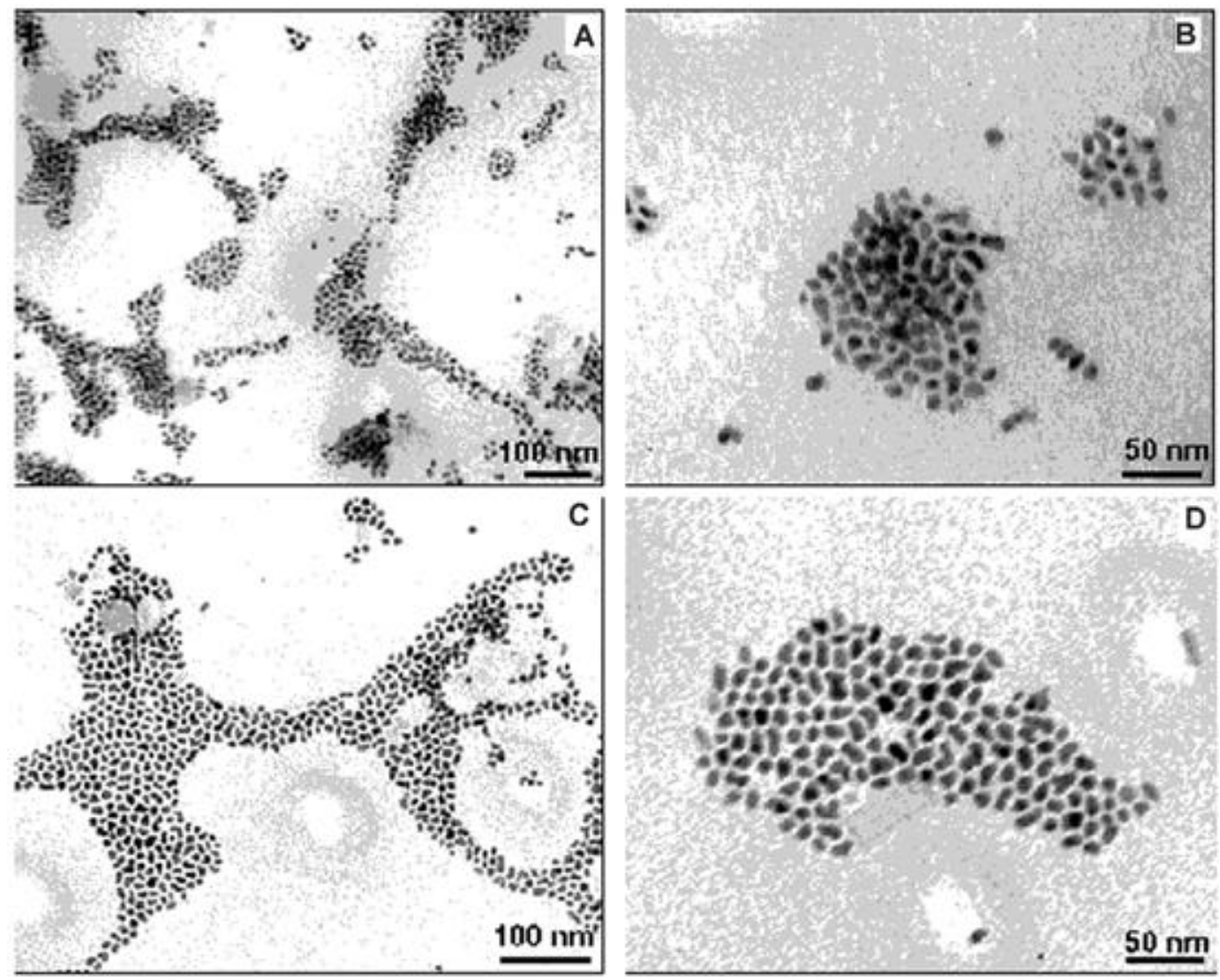

Figure 2. (A, B and C, D) Representative TEM images of ODA-capped platinum nanoparticle films formed on carbon-coated grids by solution casting from the organic phase before and after hydrogenation reaction respectively.
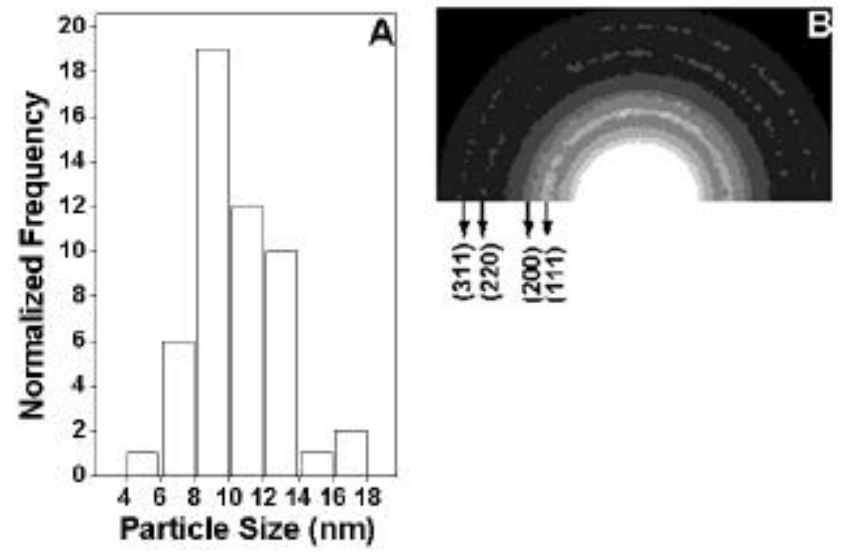

Figure 3. (A) Particle size distribution histogram measured from figure 2B. (B) Selected area electron diffraction pattern measured from $\mathrm{Pt}$ nanoparticles shown in figure $2 \mathrm{~B}$.

tes that the ODA monolayer surrounding the nanoparticles is intact.

The TEM measurements indicate that the platinum nanoparticles do not aggregate upon phase transfer into hexane and are capped with a monolayer of ODA molecules that provide sufficient hydrophobicity to the particles to accomplish their phase transfer into organic phase. Interaction of platinum nanoparticles with amines, isocyanides and polypyrrole has been reported in the literature. ${ }^{36-38}$ Perez et al have demonstrated the synthesis of 4mercaptoaniline modified platinum nanoparticles in which the hexaylamine molecules were used as intermediate labile capping agents. ${ }^{36}$ Schiffrin and coworkers synthesized alkylisocyanide-derivatized platinum nanoparticles which involve the synthesis of tetraalkylamonium bromide stabilized platinum nanoparticles by a two-phase chemical reduction method followed by place-exchange of the tetraalkylammonium ions by dodecylisocyanide molecules. ${ }^{37}$ In another report, Kim and co-workers have studied the photoelectrochemical behavior of polypyrrol modified films of $\mathrm{Pt}$ nanoparticles and showed that the photocurrent response of the film depends on the thickness of the film. ${ }^{38}$ Although a few reports have addressed the issue of surface- 
modification of $\mathrm{Pt}$ nanoparticles using thiol derivatives, the nature of the interaction in terms of its strength etc. has not been investigated in detail. The formation of ODA-capped Pt nanoparticles by the phase-transfer method reported herein provides a good system to study the interaction of alkyamines with Pt nanoparticles. In order to understand the nature and strength of bond, TGA analysis of purified powder of ODA-stabilised platinum nanoparticles was carried out. Figure 4 shows the TGA data obtained from purified powder of ODA-capped platinum nanoparticles (curve 1) and for comparison, pure ODA (curve 2). In the case of pure ODA, it is observed that there is $\approx 40 \%$ weight loss at $230^{\circ} \mathrm{C}$ which is followed by almost complete loss of the powder in the heating crucible by $600^{\circ} \mathrm{C}$ and indicates complete desorption/decomposition within this temperature interval. The ODA-Pt nano complex shows an overall weight loss of $\approx 30 \%$ at $255^{\circ} \mathrm{C}$ followed by negligible weight loss in the temperature range 434$800^{\circ} \mathrm{C}$. The weight loss at $255^{\circ} \mathrm{C}$ is due to the desorption/decomposition of the ODA molecules bound to the platinum nanoparticles. The increase in the desorption/decomposition temperature of the ODA molecules bound to platinum nanoparticles relative to free ODA molecules indicates that the interaction between the surfactant and platinum nanoparticles is quite strong. This temperature agrees reasonably well with the desorption temperatures observed in TGA analysis of alkylamine molecules covalently bound to gold nanoparticles ${ }^{28}$ indicating that the in-

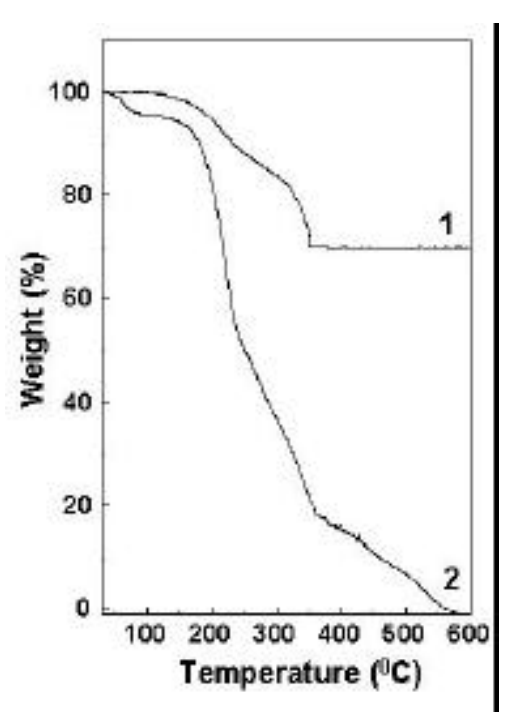

Figure 4. TGA curves of pure ODA (curve 2) and purified powder of ODA-capped platinum nanoparticles (curve 1, see text for details). teraction of alkylamines with platinum nanoparticles is of similar strength. The percentage weight contribution of the surface bound ODA molecules $(\approx 30 \%)$ is in reasonable agreement with the theoretical estimate of $\approx 24 \%$ (assuming the area occupied by ODA molecules on the nanoparticle surface as $20 \AA^{2}$ and the diameter of platinum nanoparticles to be $100 \AA$ ).

It is clear from the TGA data that the increase in desorption/decomposition temperature of ODA molecules from the surface of platinum nanoparticles is due to fairly strong interaction of amine molecules with the platinum particles, similar to that observed for alkanethiols bound to gold nanoparticles. ${ }^{28}$ In order to understand better the nature of this interaction, FTIR and XPS measurements of ODA-capped platinum nanoparticles after purification were carried out. Figures 5A and B show the FTIR spectra recorded from solution-cast films of pure ODA (curve 1) and ODA-capped platinum nanoparticles (curve 2) on Si (111) substrates in different spectral windows. A comparison of the spectra in figure $5 \mathrm{~A}$ reveals the presence of three prominent features at $\approx 2850,2920$ and $3330 \mathrm{~cm}^{-1}$ in the bare ODA film (curve 1) while the $3330 \mathrm{~cm}^{-1}$ feature disappears in the Pt-ODA film (curve 2). The $3330 \mathrm{~cm}^{-1}$ band in the bare ODA film corresponds to $\mathrm{N}-\mathrm{H}$ stretch vibrations from ODA molecules which is significantly reduced in intensity in ODA-Pt complex due to the coordination of amine molecule with the surface of platinum nanoparticles. Such a shift in the $\mathrm{N}-\mathrm{H}$ stretch vibration mode on formation of salts with anions such as $\mathrm{PtCl}_{6}^{-}$has been observed in Langmuir-Blodgett films of ODA. ${ }^{39}$ Another important observation is that the resonance at $1487 \mathrm{~cm}^{-1}$ (curve 1, figure 5B) which corresponds to the $-\mathrm{NH}_{3}$ symmetric deformation band disappears after coordination of the alkylamine molecules with the platinum nanoparticles (curve 2, figure 5B). This is additional evidence for strong complexation of amine molecules with the platinum nanoparticles. The methylene antisymmetric and symmetric vibrational modes of the hydrocarbon chains 2920 and 2850 respectively in both the native amine film and the ODA-stabilised platinum particle film (curves 1 and 2 respectively, figure 5A). Along with the fact that the methylene scissoring band occurs at $\approx 1460 \mathrm{~cm}^{-1}$ in both samples (curves 1 and 2, figure $5 \mathrm{~B}$ ) indicates that the hydrocarbon chains in the ODA monolayer surrounding the platinum particles are in a close-packed, crystalline state. ${ }^{40}$ The complete absence of $\mathrm{N}-\mathrm{H}$ stretch vibration in ODA- 


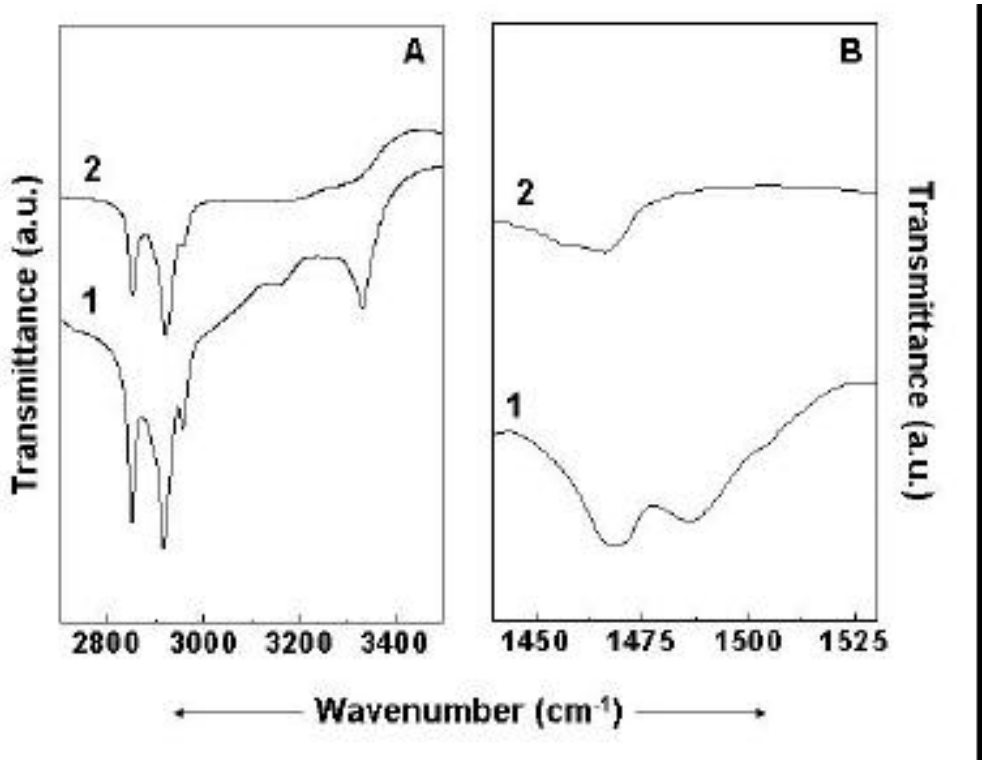

Figure 5. FTIR spectra of drop-coated films of pure octadecylamine (curve 1) and ODA-capped platinum nanoparticles (curve 2) on $\mathrm{Si}$ (111) substrates in the spectral windows 2750-3500 (figure A) and $1400-1525 \mathrm{~cm}^{-1}$ (figure B).

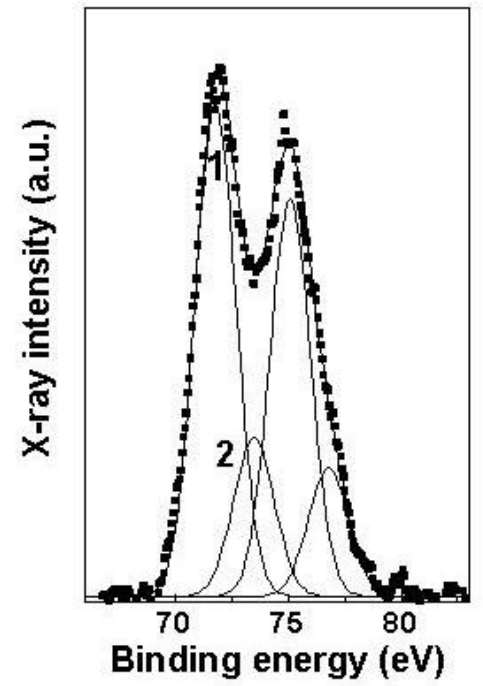

Figure 6. Pt $4 f$ core level spectrum recorded from an ODA-stabilised platinum nanoparticle film grown on a Si (111) substrate. The resolved components are shown as solid lines in the figure.

capped nanoparticles is puzzling and clearly indicates that the amine groups interact very strongly with platinum nanoparticles. It also shows that nature of bonding is very different in ODA-Pt complex that observed in simple salt formation.

A solution-cast film of ODA-capped platinum nanoparticles was formed on $\mathrm{Si}$ (111) substrates and analysed by XPS. The general scan spectrum of the film at room temperature showed the presence of $\mathrm{C} 1 s, \mathrm{~N} 1 s$ and $\mathrm{Pt} 4 f$ core levels with no evidence of impurities. The film was sufficiently thick and therefore, no signal was measured from the substrate (Si $2 p$ core level). Figure 6 shows the Pt $4 f$ core level spectrum recorded from the Pt-ODA film formed by the drop-coating technique. The spectra have been background corrected using the Shirley algorithm ${ }^{35}$ prior to curve resolution. The $\mathrm{Pt} 4 f$ spectrum could be resolved into two spin-orbit pairs (splitting $\sim 3.3 \mathrm{eV}$, identified in figure 5) with the two chemically shifted components having $4 f_{7 / 2}$ binding energies (BEs) of 72 and $73.4 \mathrm{eV}$ respectively. The low $\mathrm{BE}$ component may be assigned to electron emission from $\mathrm{Pt}(0)$ while the high $\mathrm{BE}$ component is assigned to $\mathrm{Pt}(\mathrm{II}){ }^{41}$ The $\mathrm{N} 1 s$ spectrum recorded from the ODA-capped $\mathrm{Pt}$ nanoparticles showed the presence of a single chemically distinct peak centered at $399 \mathrm{eV}$ (data not shown). This result clearly shows that there is only one mode of binding of the amine functionality with the platinum nanoparticles. However, the presence of two distinct $\mathrm{Pt}$ $4 f$ species is interesting and may be explained as follows. The low $\mathrm{BE}$ component $(\mathrm{BE}=72 \mathrm{eV})$ arises due to the metal core. The surface of the Pt nanoparticles appears to be different and is composed of a shell of $\mathrm{Pt}(\mathrm{II})$ ions. It is possible that partially reduced $\mathrm{Pt}$ ions $\left(\mathrm{PtCl}_{4}^{2-}\right)$ cap the surface of the metal core and electrostatically complex with protonated amine 
groups during phase transfer. Indeed, phase transfer of the Pt nanoparticles from water to hexane could not be achieved when the $\mathrm{pH}$ of the hydrosol was adjusted to 12. At this $\mathrm{pH}$, the amine groups of ODA are unprotonated and incapable of electrostatically complexing with the negatively charged Pt nanoparticle surface thereby inhibiting phase transfer.

The interaction of amine molecule with platinum is well known in the literature. Venand and coworkers have shown through NMR studies of platinum complexes such as cis- $\left[\mathrm{PtCl}_{2} \mathrm{~A}_{2}\right]$, trans- $\left[\mathrm{PtCl}_{2}\right.$ $\left.\mathrm{A}_{2}\right]$, cis- $\left[\mathrm{PtBr}_{2} \mathrm{~A}_{2}\right]$, trans-[ $\left[\mathrm{PtBr}_{2} \mathrm{~A}_{2}\right]$, cis- $\left[\mathrm{PtCl}_{4} \mathrm{~A}_{2}\right]$, cis-[ $\left[\mathrm{PtBr}_{4} \mathrm{~A}_{2}\right],\left[\mathrm{PtBrA}_{3}\right] \mathrm{Br},\left[\mathrm{PtA}_{4}\right] \mathrm{Br}_{2}$ where $\mathrm{A}=n$ dodecylamine $^{42}$ that the coupling constant $(J){ }^{195} \mathrm{Pt}$ ${ }^{15} \mathrm{~N}$ proportional to the $s$ character involve in the bonding orbital. In another report Romeo et al have performed kinetic studies of the nucleophilic substitution of 5-aminoquinoline (5-Aq) and diethyl sulphide from the complexes cis- $\left[\mathrm{PtPh}_{2}(\mathrm{CO})(\mathrm{L})\right](\mathrm{L}=$ $5-\mathrm{Aq}$ and $\mathrm{SEt}_{2}$ ) by an extended series of aliphatic amines of comparable basicity $(\mathrm{pK},=10.6 \pm 0.4)$ and of different steric hindrance. ${ }^{43}$ These reports indicate the strong affinity of amine molecules for $\mathrm{Pt}(\mathrm{II}) / \mathrm{Pt}(\mathrm{IV})$ and are in agreement with the TGA, FTRI and XPS data discussed earlier.

It is clear that complexation of ODA molecules with the Pt nanoparticles enables their phase transfer into hexane. Given the importance of Pt in catalysis, a relevant question to ask would be whether the alkyamine capped Pt nanoparticles would continue to be catalytically active. We measured the catalytic activity of the hydrophobized platinum nanoparticles in a simple hydrogenation reactions involving the conversion of styrene to ethylbenzene and cyclohexene to cyclohexane. These reactions were studied in a $100 \mathrm{ml}$ reactor (supplied by Parr Instruments Company, USA). Analysis of the initial and final samples were done using gas chromatography (Varian 3400; CP-SIL 8CB Column; with a $30 \mathrm{~m}$ long and $0.53 \mathrm{~mm}$ inner diameter) having the conditions: initial column temp. $80^{\circ} \mathrm{C}: 80-180^{\circ} \mathrm{C}$ at the rate of $3^{\circ} \mathrm{C}$ per min: $180-240^{\circ} \mathrm{C}$ at the rate of $10^{\circ} \mathrm{C}$ per min. Reduction of styrene $(10 \mathrm{mmol})$ to ethyl benzene and cyclohexene $(10 \mathrm{mmol})$ to cyclohexane was carried out in methanol and hexane respectively. The GC analysis showed $87 \%$ conversion of styrene to ethyl benzene with $100 \%$ selectivity (TOF $19,683 \mathrm{~h}^{-1}$ ). The platinum nanoparticle catalyst also worked excellently in the hydrogenation of a non-aromatic substrate such as cyclohexene giving $91 \%$ conversion from to cyclohexane with $98.8 \%$ selectivity (TOF
$20,588 \mathrm{~h}^{-1}$ ). Both the reactions were conducted at $200 \mathrm{psig}$ hydrogen pressure for $2 \mathrm{~h}$ at $100^{\circ} \mathrm{C}$. Further evidence that the ODA monolayer surrounding the particles is intact after the hydrogenation reaction was provided by Fourier transform infrared spectroscopy analysis of the particles that showed the presence of the characteristic $\mathrm{N}-\mathrm{H}$ stretch vibrational modes (data not shown).

In conclusion, the phase transfer of aqueous platinum nanoparticles into hexane using octadecylamine molecules present in the organic phase has been demonstrated. The fatty amine-capped platinum particles are extremely stable, could be easily precipitated out of solution and redissolved in different organic solvents without significant variation in the particle size. The hydrophobized Pt nanoparticles are highly active and catalyse the hydrogenation of styrene to ethylbenzene and cyclohexene to cyclohexane with almost $98 \%$ selectivity.

\section{Acknowledgements}

AK would like to thank the Council for Scientific and Industrial Research, New Delhi for a fellowship. This work was partially supported by a grant from the Department of Science and Technology (DST), Government of India and we gratefully acknowledge it.

\section{References}

1. Hoffman A J, Mills G, Yee H and Hoffman M R 1992 J. Phys. Chem. 965546

2. Mukherjee P, Patra C R, Ghosh A, Kumar R and Sastry M 2002 Chem. Mater. 141678

3. Colvin V L, Schlamp M C and Alivisatos A P 1994 Nature (London) 370354

4. Baschong W and Wrigley N G 1990 J. Electron. Microsc. Technol. 14313

5. Elghanian R, Storhoff J J, Mucic R C, Letsinger R L and Mirkin C A 1997 Science 2771078

6. Turkevich J, Garton G and Stevenson P C $1954 J$. Colloid Sci. 926

7. Handley D A 1989 Colloidal gold: Principles, methods and applications (ed.) M A Hayat (San Diego: Academic Press) vol. 1, ch. 2

8. Duff D G, Baiker A and Edwards P P 1993 Langmuir 92301

9. Henglein A 1999 Langmuir 156738

10. Gachard E, Remita H, Khatouri J, Keita B, Nadjo L and Belloni J 1998 New J. Chem. 1257

11. Mizukoshi Y, Fujimoto T, Nagata Y, Oshima R and Maeda Y 2000 J. Phys. Chem. B104 6028 
12. Mukherjee P, Ahmad A, Mandal D, Senapati S, Sainkar S R, Khan M I, Ramani R, Parischa R, Ajayakumar P V, Alam M, Sastry M and Kumar R 2001 Angew. Chem., Int. Ed. 403585

13. Mukherjee P, Senapati S, Mandal D, Ahmad A, Khan M I, Kumar R and Sastry M 2002 ChemBioChem 3461

14. Brust M, Walker M, Bethell D, Schiffrin D J and Whyman R 1994 J. Chem. Soc., Chem. Commun. 801

15. Nuzzo R G and Allara D L 1993 J. Am. Chem. Soc. 1054481

16. Selvakannan P R, Mandal S, Pasricha R, Adyanthaya S D and Sastry M 2002 Chem. Commun. 1334

17. Leff D V, Ohara P C, Heath J C and Gelbart W M 1995 J. Phys. Chem. 997036

18. Cliffel D E, Zamborini F P, Gross S M and Murray R W 2000 Langmuir 169699

19. Shon Y-S, Wuelfling W P and Murray R W 2001 Langmuir 171255

20. Underwood S and Mulvaney P 1994 Langmuir 10 3427

21. Sarathy K V, Kulkarni G U and Rao C N R 1997 Chem. Commun. 537

22. Sarathy K V, Raina G, Yadav R T, Kulkarni G U and Rao C N R 1997 J. Phys. Chem. B101 9876

23. Wang W, Efrima S and Regev O 1998 Langmuir 14 602

24. Liz-Marzan L M and Lado-Tourino 1996 Langmuir 123585

25. Sastry M, Kumar A and Mukherjee P 2001 Coll. Surf. A. 181255

26. Lala N, Lalbegi S P, Adyanthaya S D and Sastry M 2001 Langmuir 173766
27. Kumar A, Mandale A B and Sastry M 2000 Langmuir 169299

28. Leff D V, Brandt L and Heath J R 1996 Langmuir 12 4723

29. Brown L O and Hutchison J E $1999 \mathrm{~J}$. Am. Chem. Soc. 121882

30. Duff D G and Edwards P P 1995 J. Phys. Chem. 99 15934

31. Chen C W, Tano D and Akashi M 1999 Colloid Polym. Sci. 277488

32. Dalmia A, Lineken C L and Savinell R F 1998 J. Colloid Interface Sci. 205535

33. Ahmadi T S, Wang Z L, Green T C, Henglein A and Ei-Sayed M A 1996 Science 2721924

34. Patil V, Malvankar R B and Sastry M 1999 Langmuir 158197

35. Shirley D A 1972 Phys. Rev. B5 4709

36. Perez H, Pradeau J-P, Albouy P-A and Perez-Omil J 1999 Chem. Mater. 113460

37. Horswell S L, Kiely C J, O'Neil I A and Schiffrin D J 1999 J. Am. Chem. Soc. 1215573

38. Kim H and Chang W 1999 Synth. Met. 101150

39. Bardosava M, Tredgold R H and Ali-Adib Z 1995 Langmuir 111273

40. Hostetler M J, Stokes J J and Murray R W 1996 Langmuir 123604

41. Walter J, Nishioka M and Hara S 2001 Chem. Mater. 131828

42. Pregosin P S, Omura H and Venand L M 1973 J. Am. Chem. Soc. 952047

43. Romeo R, Arena G, Scolaro L M, Plutino M R, Bruno G and Nicol F 1994 Inorg. Chem. 334029 